\title{
The right to refuse unwanted citations: rethinking the culture of science around the citation
}

\author{
Jaime A. Teixeira da Silva ${ }^{1} \cdot$ Quan-Hoang Vuong ${ }^{2,3}$ \\ Received: 14 February 2021 / Accepted: 17 March 2021 / Published online: 8 May 2021 \\ (c) Akadémiai Kiadó, Budapest, Hungary 2021
}

\begin{abstract}
Logically, and by most common standards, academics would be pleased to be cited, considering it a form of recognition of their intellect. In return, especially those with high citation counts, such as Clarivate Analytics' Highly Cited Researchers, can benefit through peer recognition, rewards, funding, securing a better position, or expanding a collaborative network. Despite known and untold benefits, one issue has not been discussed: the right to refuse to be cited or the right to refuse a citation. Academics might not want to be cited by papers published in truly predatory journals, papers with false authors, or sting papers with falsified elements that employ underhanded ethical tactics. Currently, academics generally have the freedom to select where they publish their findings and choose studies they cite, so it is highly probable that requests to remove citations or refuse citations might never become formal publishing policy. Nonetheless, this academic discussion is worth having as valid and invalid literature increasingly gets mixed through citations, and as the grey zone between predatory/non-predatory and scholarly/unscholarly becomes increasingly difficult to distinguish.
\end{abstract}

Keywords Author- and journal-based metrics · Citation boosting and manipulation . Predatory publishing

Jaime A. Teixeira da Silva

jaimetex@yahoo.com

Quan-Hoang Vuong

hoang.vuongquan@phenikaa-uni.edu.vn; qvuong@ulb.ac.be

1 Independent researcher, Ikenobe 3011-2, P. O. Box 7, Miki-cho, Kagawa-ken 761-0799, Japan

2 Centre for Interdisciplinary Social Research, Phenikaa University, Ha Dong District,

Hanoi 100803, Viet Nam

3 Centre Emile Bernheim, Université Libre de Bruxelles, 1050 Brussels, Belgium 
Dear Scientometrics Editors,

\section{Introduction}

While the vast majority of the academic conversation surrounding citations revolves around the need to be cited, the advantage of citations to authors or journals (Patience et al., 2017), or the abuse or manipulation of citations (Baccini et al., 2019; Fong \& Wilhite, 2017), the question "what if I don't want my work to be cited?" has rarely been asked in the academic literature. This is likely because academics, including editors, tend to perceive citations, excluding citation rings (Fister et al., 2016) and citation doping and stacking (Ball, 2002; Van Noorden \& Chawla, 2019), as a form of intellectual reward or recognition. In fact, an entire competitive field of vanity publishing dedicates itself to rewarding high citation levels, namely Clarivate Analytics' Highly Cited Researchers (Teixeira da Silva \& Bernès, 2018). The question being raised here is also obscure because researchers themselves tend to be either so preoccupied with their work or craving citations that they rarely think whether being cited can be undesirable. As academics and publishers work together to improve publishing practices as well as to become more transparent and prone to self-correction (Vuong, 2020), a nuanced discussion on citations should thus take into account not only the reform of citation metrics and citation-based scientific funding and promotion but also the perspectives and rights of cited authors.

\section{Some citations are "more equal than others"}

Reasons for not wanting to be cited pertain to an academic's desire not to be associated with inaccurate, irrelevant, and/or poor-quality research. Table 1 lays out three major circumstances for unwanted citations concerning the characteristics of the citing author(s), the representation of the citation, and the presence of the citation. In particular, published authors would like to be far removed from citing any authors that are involved in unethical (Aa), fake, or fraudulent practices (Ab), or whose articles have been retracted from the literature (Ac). Additionally, no authors would ever want their original ideas to be misinterpreted, distorted $(\mathrm{Ba})$, or cited in a completely irrelevant manner $(\mathrm{Bb})$. Skewed citation practices, which include the citing of inappropriate and inaccurate papers, have been noted as quite common in research on marine biology (Todd et al., 2010), ecological science (Todd et al., 2007), and biomedicine (Karabulut, 2016).

In many cases of "lazy" or thoughtless citation, a citing author might use a reference without having read the source study and cite it in a way that is both irrelevant and shallow to the literature at hand (Ball, 2002). While this might not constitute academic misconduct,

Table 1 Some characteristics of unwanted citations

\begin{tabular}{llll}
\hline & A & B & C \\
& Citing authors & Representation of citation & Presence of citation \\
\hline $\mathrm{a}$ & Unethical sting operation & Misinterpretation & Valid journal \\
$\mathrm{b}$ & Fake, false authors & Irrelevance & Low-quality journal \\
$\mathrm{c}$ & Authors of the retracted paper & Correct interpretation & Fake, predatory journal \\
\hline
\end{tabular}


it is nonetheless a matter of negligence. It is important to add that an author may also not like it, even when their work is accurately represented, but is associated with a paper published in a low-quality (a combination of $\mathrm{Bc}$ and $\mathrm{Cb}$ ) and/or a fake, predatory journal (Bc and $\mathrm{Cc}$ ). In another circumstance, a citation might also become undesirable when it appears in a retracted paper, regardless of being published in a valid journal (Ac and $\mathrm{Ca}$ ). This examination shows that understanding an author's motivations for unwanted citations requires taking into account a complex set of factors and circumstances.

This paper raises questions about unwanted citations in a paper from a low-quality $(\mathrm{Cb})$ or predatory journal $(\mathrm{Cc})$. The risks of unwanted citations in predatory venues are potentially amplified during the new coronavirus (COVID-19) pandemic (Teixeira da Silva, 2020a; Vervoort et al., 2020). A recent review of 367 articles published in potentially predatory journals revealed how misinformation, such as the efficacy of homeopathic treatments in combating COVID-19, may be spread and could carry serious health consequences (Vervoort et al., 2020). The concerns are therefore legitimate in consideration of the following aspects about truly predatory journals, or publishers ${ }^{1}$ : (1) little or no quality control, either as peer review or editorial handling, exists, so any literature is approved and published without critical scrutiny; (2) academic work might be misrepresented, miscited, or erroneously cited, i.e., the citation might be used simply to give a reference list and "academic" feel, but not necessarily support the claims being made. In such cases, would a request by an academic — who is cited — not to be cited be unreasonable?

\section{The right not to be cited?}

In theory, an academic should have such a right (Al-Khatib \& Teixeira da Silva, 2017). Still, it would be impossible to exercise that right in practice because it would have to involve greater weight than editorial independence. Authors rarely have such leverage. In some published forms, like preprints, where follow-up versions allow for corrections of the paper (Coudert, 2020; Kaiser, 2017), it would be possible to exercise this right by requesting authors not to cite their work or by putting forward arguments to editors to exclude select citations. In exceptional cases, at least in theory, an academic that does not wish their intellect to be misinterpreted or represented in a low-quality or predatory journal or a fake paper (Bohannon, 2015) could approach the editors during post-publication and request that their citation be removed, or replaced. This is theoretically possible if the editors agree to issue a corrigendum or retract and replace a paper to accommodate this extraordinary request (Bordignon, 2020). A small caveat to this possibility is that currently, academics, including thought leaders in the field of "predatory publishing," are unable to clearly distinguish all predatory from non-predatory entities (Grudniewicz et al., 2019), except for clear cases, such as OMICS International (Manley, 2019). However, the fact remains that a wide grey zone of ambivalence exists (Teixeira da Silva, 2020b).

Sting papers in academic publishing often involve fake or falsified elements, whether these be the author's name, affiliation, email, or content, and are unethical in nature, independent of their objective (Teixeira da Silva, 2021). If a sting paper cites an author's paper, and the author identifies this citation, can they request the editor to remove their citation so as not to be associated with the sting paper? In essence, if a sting paper were to be retracted on ethical grounds, then the citations would automatically be annulled, in

\footnotetext{
1 We recognize that such characteristics are becoming increasingly prevalent in ranked and indexed journals, even those with metrics.
} 
principle (Anderson, 2019). Currently, no ethics guidelines exist pertaining to the selective retraction or withdrawal of a single or select citations from a paper's reference list.

One complex issue is associated with this right, namely citation and selection bias (Duyx et al., 2017). Specifically, an academic may conscientiously and erroneously cite, or purposefully select against, rival academics' valid literature whom they do not wish to cite. Empirical evidence has even pointed out that, in the field of neuroscience, male authors tend to be cited more than female authors, an imbalance that is worsening over time due to the overrepresentation of male researchers (Dworkin et al., 2020). Citation bias, a problem detected in science as early as the 1980s and 1990s (Beach, 1984; Paris et al., 1998), is one form of a conflict of interest termed snub publishing (Teixeira da Silva, 2014). In principle, it is the academic responsibility of peers and editors to detect and moderate such a bias (Ekmekci, 2017; Teixeira da Silva \& Dobránszki, 2018; Thornton \& Lee, 2000; Tóth, 2020). Ultimately, such adjustments or selection bias would impact or skew author- and journal-based metrics, as well as citation-related databases and/or indexes such as Scopus, Google Scholar, PubMed, or Web of Science (Doğan et al., 2016; Franceschini et al., 2012). Given that the calculation of the journal impact factor (JIF) is largely concerned with the count of citations, it is possible to game the system by selectively citing papers from a certain journal (Biagioli, 2016; Kapeller, 2010). Questions about the validity and reliability of JIF aside, citation bias-which includes a preference for positive or statistically significant findings, gender imbalance in citation practice, excessive citations, and unwarranted self-citations-is clearly a contributor to the misuse of journal metrics.

\section{Conclusion}

This paper highlights several troubling concerns about current citing practices: being cited in a low-quality or predatory journal, being cited by fake authors or being cited inaccurately. Thus, we argue for an author's right to refuse undesirable citations. In practice, this right is very difficult to exercise under the current hyper-competitive and resource-strained climate in academia. To exercise this right would require a seismic change in the culture of science around the citation, which starts with rethinking the current obsession with journal metrics, JIF, and citation rankings as such a mindset might only perpetuate bad practices in science (Biagioli, 2016). There is a difference between shoddy and high-quality science.

Similarly, there is a difference between a shoddy and a high-quality citation. Eliminating shoddy citations can increase science's quality, reducing the cost of doing science, and increasing the public's trust in academia (Vuong, 2018). The challenge moving forward will be to clearly distinguish both categories of citations, especially as the number of retractions from journals that were traditionally perceived to be high-quality (e.g., with peer review, a JIF, PubMed-indexed, etc.) become mixed in the published literature. As equally as sound bites can destroy a public person's reputation, problematic citations can certainly harm a scientist's reputation. It is now time to start a conversation about how to reform this aspect of our citing practice.

Authors' contributions The authors contributed equally to the intellectual discussion underlying this paper, literature exploration, writing, reviews, editing, and accepting responsibility for the content and interpretation.

Funding This research was not funded. 


\section{Declarations}

Conflicts of interest The authors declare no relevant conflicts of interest relevant to this topic.

\section{References}

Al-Khatib, A., \& Teixeira da Silva, J. A. (2017). What rights do authors have? Science and Engineering Ethics, 23(3), 947-949. https://doi.org/10.1007/s11948-016-9808-8.

Anderson, R. (2019). Citation contamination: references to predatory journals in the legitimate scientific literature. The Scholarly Kitchen. Retrieved from https://scholarlykitchen.sspnet.org/2019/10/28/citat ion-contamination-references-to-predatory-journals-in-the-legitimate-scientific-literature/. March 13, 2021.

Baccini, A., De Nicolao, G., \& Petrovich, E. (2019). Citation gaming induced by bibliometric evaluation: A country-level comparative analysis. PLoS ONE, 14(9), e0221212. https://doi.org/10.1371/journal.pone. 0221212 .

Ball, P. (2002). Paper trail reveals references go unread by citing authors. Nature, 420(6916), 594. https:// doi.org/10.1038/420594a.

Beach, L. R. (1984). The citation bias: Fad and fashion in the judgment and decision literature. American Psychologist, 39(1), 75-78. https://doi.org/10.1037/0003-066X.39.1.75.

Biagioli, M. (2016). Watch out for cheats in citation game. Nature, 535, 201. https://doi.org/10.1038/53520 $1 \mathrm{a}$.

Bohannon, J. (2015). Hoax-detecting software spots fake papers. Science, 348(6230), 18-19. https://doi.org/ $10.1126 /$ science.348.6230.18.

Bordignon, F. (2020). Self-correction of science: A comparative study of negative citations and post-publication peer review. Scientometrics, 124(2), 1225-1239. https://doi.org/10.1007/s11192-020-03536-Z.

Coudert, F.-X. (2020). The rise of preprints in chemistry. Nature Chemistry, 12(6), 499-502. https://doi.org/ 10.1038/s41557-020-0477-5.

Doğan, G., Şencan, İ, \& Tonta, Y. (2016). Does dirty data affect google scholar citations? Proceedings of the Association for Information Science and Technology, 53(1), 1-4. https://doi.org/10.1002/pra2. 2016.14505301098.

Duyx, B., Urlings, M. J. E., Swaen, G. M. H., Bouter, L. M., \& Zeegers, M. P. (2017). Scientific citations favor positive results: A systematic review and meta-analysis. Journal of Clinical Epidemiology, 88, 92-101. https://doi.org/10.1016/j.jclinepi.2017.06.002.

Dworkin, J. D., Linn, K. A., Teich, E. G., Zurn, P., Shinohara, R. T., \& Bassett, D. S. (2020). The extent and drivers of gender imbalance in neuroscience reference lists. Nature Neuroscience, 23(8), 918-926. https://doi.org/10.1038/s41593-020-0658-y.

Ekmekci, P. E. (2017). An increasing problem in publication ethics: Publication bias and editors' role in avoiding it. Medicine, Health Care and Philosophy, 20(2), 171-178. https://doi.org/10.1007/ s11019-017-9767-0.

Fister, I., Fister, I., \& Perc, M. (2016). Toward the discovery of citation cartels in citation networks. Frontiers in Physics, 4, 49. https://doi.org/10.3389/fphy.2016.00049.

Fong, E. A., \& Wilhite, A. W. (2017). Authorship and citation manipulation in academic research. PLoS ONE, 12(12), e0187394-e0187394. https://doi.org/10.1371/journal.pone.0187394.

Franceschini, F., Maisano, D., \& Mastrogiacomo, L. (2012). The effect of database dirty data on h-index calculation. Scientometrics, 95(3), 1179-1188. https://doi.org/10.1007/s11192-012-0871-x.

Grudniewicz, A., Moher, D., Cobey, K. D., Bryson, G. L., Cukier, S., Allen, K., Ardern, C., Balcom, L., Barros, T., Berger, M., Ciro, J. B., Cugusi, L., Donaldson, M. R., Egger, M., Graham, I. D., Hodgkinson, M., Khan, K. M., Mabizela, M., Manca, A.,... Lalu, M. M. (2019). Predatory journals: No definition, no defence. Nature, 576, 210-212. https://doi.org/10.1038/d41586-019-03759-y.

Kaiser, J. (2017). The preprint dilemma. Science, 357(6358), 1344-1349. https://doi.org/10.1126/science. 357.6358.1344.

Kapeller, J. (2010). Citation metrics: Serious drawbacks, perverse incentives, and strategic options for heterodox economics. American Journal of Economics and Sociology, 69(5), 1376-1408. https://doi.org/ 10.1111/j.1536-7150.2010.00750.x.

Karabulut, N. (2016). Inaccurate citations in biomedical journalism: Effect on the impact factor of the American Journal of Roentgenology. American Journal of Roentgenology, 208(3), 472-474. https:// doi.org/10.2214/AJR.16.16984. 
Manley, S. (2019). Predatory journals on trial: Allegations, responses, and lessons for scholarly publishing from FTC v. OMICS. Journal of Scholarly Publishing, 50(3), 183-200. https://doi.org/10.3138/jsp. 50.3.02.

Paris, G., De Leo, G., Menozzi, P., \& Gatto, M. (1998). Region-based citation bias in science. Nature, 396(6708), 210-210. https://doi.org/10.1038/24249.

Patience, G. S., Patience, C. A., Blais, B., \& Bertrand, F. (2017). Citation analysis of scientific categories. Heliyon, 3(5), e00300-e00300. https://doi.org/10.1016/j.heliyon.2017.e00300.

Teixeira da Silva, J. A. (2014). Snub publishing: Evidence from the Anthurium literature. Publishing Research Quarterly, 30(1), 166-178. https://doi.org/10.1007/s12109-014-9355-6.

Teixeira da Silva, J. A. (2020a). An alert to COVID-19 literature in predatory publishing venues. Journal of Academic Librarianship, 46(5), 102187-102187. https://doi.org/10.1016/j.acalib.2020.102187.

Teixeira da Silva, J. A. (2020b). Is there a clear division between predatory and low-quality journals and publishers? Journal of the Royal College of Physicians of Edinburgh, 50(4), 458-459. https://doi.org/ $10.4997 /$ jrcpe. 2020.427.

Teixeira da Silva, J. A. (2021). Assessing the ethics of stings, including from the prism of guidelines by ethics-promoting organizations (COPE, ICMJE, CSE). Publishing Research Quarterly, 37(1), 90-98. https://doi.org/10.1007/s12109-021-09784-y.

Teixeira da Silva, J. A., \& Bernès, S. (2018). Clarivate Analytics: Continued omnia vanitas impact factor culture. Science and Engineering Ethics, 24(1), 291-297. https://doi.org/10.1007/s11948-017-9873-7.

Teixeira da Silva, J. A., \& Dobránszki, J. (2018). Editors moving forward: Stick to academic basics, maximize transparency and respect, and enforce the rules. Recenti Progressi in Medicina, 109(5), 263-266. https://doi.org/10.1701/2902.29244.

Thornton, A., \& Lee, P. (2000). Publication bias in meta-analysis: Its causes and consequences. Journal of Clinical Epidemiology, 53(2), 207-216. https://doi.org/10.1016/S0895-4356(99)00161-4.

Todd, P. A., Guest, J. R., Lu, J., \& Chou, L. M. (2010). One in four citations in marine biology papers is inappropriate. Marine Ecology Progress Series, 408, 299-303. https://doi.org/10.3354/meps08587.

Todd, P. A., Yeo, D. C., Li, D., \& Ladle, R. J. (2007). Citing practices in ecology: Can we believe our own words? Oikos, 116(9), 1599-1601. https://doi.org/10.1111/j.0030-1299.2007.15992.x.

Tóth, J. (2020). Blind myself: Simple steps for editors and software providers to take against affiliation bias. Science and Engineering Ethics, 26(3), 1875-1877. https://doi.org/10.1007/s11948-019-00128-w.

Van Noorden, R., \& Chawla, D. S. (2019). Hundreds of extreme self-citing scientists revealed in new database. Nature, 572, 578-579. https://doi.org/10.1038/d41586-019-02479-7.

Vervoort, D., Ma, X., \& Shrime, M. G. (2020). Money down the drain: predatory publishing in the COVID-19 era. Canadian Journal of Public Health, 111(5), 665-666. https://doi.org/10.17269/ s41997-020-00411-5.

Vuong, Q.-H. (2018). The (ir)rational consideration of the cost of science in transition economies. Nature Human Behaviour, 2(1), 5-5. https://doi.org/10.1038/s41562-017-0281-4.

Vuong, Q. H. (2020). Reform retractions to make them more transparent. Nature, 582(7811), 149. https:// doi.org/10.1038/d41586-020-01694-x. 